\title{
Effectiveness Evaluation Method for Digital Logistics Equipment System of Systems
}

\author{
Xiaolei Zheng \\ The Logistics College, Beijing, P.R. China \\ Ji Ren \\ The Equipment Demonstration Center, Beijing, P.R. China
}

\begin{abstract}
Based on grey theory, integrating Analytical Hierarchy Process and information entropy, this paper put for-ward an effectiveness evaluation method of digital Logistics equipment system of systems; the index of digital Logistics equipment system of systems was established, the method of effectiveness method based on the index was discussed; this method is of impersonality and in reason, which provided a simple and practical method for effectiveness evaluation for digital Logistics equipment system of systems.

KEYWORD: Grey theory; Analytical hierarchy process; Information entropy; Digital Logistics equipment system of systems; Effectiveness evaluation;
\end{abstract}

\section{INTRODUCTION}

Supported by integrative information systems, Digital Logistics Equipment System of Systems is composed of digital weapons or equipment for detection, hitting or protection, and logistical support systems as an integral combat equipment system of systems through the step-by-step connection of combat components by tactical internet. In this way, the antagonism between platforms could be transformed as that between systems of systems, which is exactly the necessary development orientation of future army (CJCS2009).

At present, Digital Logistics Equipment System of Systems has been demonstrated preliminarily. With the new features and new requisitions of the overall performance of information-based weapons and equipment, the requirement for analyzing and assessing Digital Logistics Equipment System of Systems combat effectiveness becomes more urgent. And this provides advantageous foundation for the optimization of digital weapons and equipment systems in army (Lizotte et al. 2004, TTCP 2004).

For the purpose of assessing the combat effectiveness of Digital Logistics Equipment System of Systems from both qualitative and quantitative perspectives to provide reference support for decisionmakers, this paper comprehensively applies the calculative ideas of AHP(Maier 1998), Grey Correlation Degree (Sage \& Cuppan 2001)and Information Entropy(DeLaurentis 2005) and designs a simple and convenient method.

\section{CONSTRUCTION OF TARGET SYSTEM}

As shown in Figure1, considering the combat process and combat mode between information-based systems of systems, a target system for evaluating the effectiveness of Digital Logistics Equipment System of Systems is constructed:

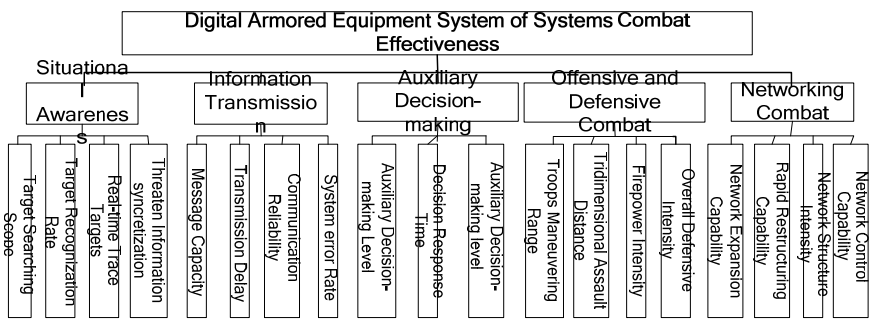

Figure 1. Target System for Digital Logistics Equipment System of Systems Combat Effectiveness Evaluation

In the combat between systems of systems, Digital Logistics Equipment System of Systems focuses on the capability of situational awareness, information transmission, auxiliary decision-making and networking combat. Thereby, these capabilities are regarded as the top target with the capability of offensive and defensive combat. Under this level are concrete and accurate quantitative indices. When it comes to the targets which can hardly be quantified, such as networking combat capability, expert scoring method can be adopted to obtain the qualitative values of the indices. 


\section{STANDARDIZATION OF INDICES}

Indices can be qualitative or quantitative, which manifest the feature of different degrees and contradiction. Therefore, it is impossible to synthetically calculate all these indices values unless they are normalized and standardized. Qualitative indices tend to gain their values by expert qualitative judgment and quantification, while quantitative indices are evaluated through experiment statistics, field analysis, and report analysis (Sage \&Cuppan 2001, DeLaurentis 2005, Ramon et al. 2009, Griendling\&Mavris 2011).

\subsection{The normalization of qualitative indices}

So as to simplify the process, the method of quantizationscales is adopted to map the qualitative evaluation to the values between 0 and 1 , as shown in Table I. It is advised to consider 5 to 9 grades quantification in the qualitative judgment, 9 to be the best if possible. This is theoretically and practically reasonable because average persons could tell the difference from Grade 5 to 9 clearly.

Table 1 THE QUANTITIVE SCALESOF QUALITIVE INDICES

\begin{tabular}{|c|c|c|c|c|c|c|c|c|c|}
\hline Scores & 0.1 & 0.2 & 0.3 & 0.4 & 0.5 & 0.6 & 0.7 & 0.8 & 0.9 \\
\hline Grade 9 & worst & worse & bad & sub-average & average & fine & good & better & best \\
\hline Grade 7 & worst & worse & bad & & average & fine & good & best \\
\hline Grade 5 & worst & & bad & & average & & good & best \\
\hline
\end{tabular}

What's more, human words could be quantified as fuzzy values using the scale quantification method, usually including triangular fuzzy numbers and trapezoidal fuzzy numbers (DeLaurentis 2005), which avoids the loss of fuzzy information effectively but tends to be a complex calculation process, especially the final sequence. In this article, the quantization scale method is utilized to make the approach simple and practical.

\subsection{The standardization of quantitative indices}

The following situations exist generally in the assessment process of Logistics equipment system of systems effectiveness: (1) different degree, which means that all indices have different dimensions making it inconvenient to compare; (2) different variance scopes, even different order of magnitude, which make it complex to calculate; (3) different antagonism characteristics, implying that some indices could be as large as possible, while others might be as small as possible (TTCP 2004).

Therefore, as for a problem of effectiveness assessment with known indices matrix, it is necessary to standardize (normalize) it; that is to transform the index value into another one in $[0,1]$ by some certain mathematical manipulation. Regarding the types of indices presented in literature (Biltgen\&Mavris 2007), the following indices standardization methods could be used to standardize quantitative indices:

(1) Maximum Indices:

$$
r_{i}=x_{i, j}-\frac{\min _{i} x_{i, j}}{\max _{i} x_{i, j}}-\min _{i} x_{i, j},
$$

Where $r_{i}$ denotes the corresponding index while $x_{i, j}$ represents the bottom index.

(2) Maximum Indices:

$$
r_{i}=\max x_{i, j}-\frac{x_{i, j}}{\max _{i} x_{i, j}}-\min _{i} x_{i, j}
$$

(3) Regular Indices:

$$
r_{i, j}= \begin{cases}1, & x_{i, j}=x_{i}^{*} \\ 1-\frac{\left|x_{i, j}-x_{i}^{*}\right|}{\max _{i}\left|x_{i, j}-x_{i}^{*}\right|} & x_{i, j} \neq x_{i}^{*}\end{cases}
$$

$x_{i}^{*}$ is the optimum value of $x_{i}$ which also denotes the bottom index.

(4) Interval Indices:

$$
r_{i, j}=\left\{\begin{array}{lc}
1-\frac{q_{i, 1}-x_{i, j}}{\max \left\{\min _{i} x_{i, j}-q_{i, 1}, \max _{i} x_{i, j}-q_{i, 2}\right\}} & x_{i, j}<q_{i, 1} \\
1-\frac{x_{i, j}-q_{i, 2}}{\max \left\{\min _{i} x_{i, j}-q_{i, 1}, \max _{i} x_{i, j}-q_{i, 2}\right\}} & x_{i, j} \in\left[q_{i, 1}, q_{i, 2}\right]
\end{array}\right.
$$

$\left[q_{1}, q_{2}\right]$ is the optimum interval value of the interval indices.

\section{EFFECTIVENESS EVALUATION BASED ON INFORMATION ENTROPY AND GREY CORRELATION DEGREE}

The application of Grey Correlation Degree concept is a relative sorting analysis in effectiveness evaluation, and accordingly, calculating the weights of all the indices plays a significant role in this process. In the practical application, the weights can be determined by AHP or the concept of Information Entropy.

Suppose that there are $\mathrm{n}$ planning schemes of digital Logistics equipment system of systems for evaluation, and $x_{i}=\left(x_{i 1}, x_{i 2}, \ldots, x_{i 17}\right)$ represents the bottom indices set of system $\mathrm{i}$. To simplify the comparison, the 
original indices should be standardized as values between 0 and 1.

\subsection{Grey correlation coefficient and correlation degree}

The ideal values of standardized indices $r^{*}=\left(r_{1}{ }^{*}, r_{2}{ }^{*}, \ldots, r_{17}{ }^{*}\right)$ are taken as the indices values of this system of systems as a reference, and $r=\left(r_{1}, r_{2}, \ldots, r_{17}\right)$ are the set for comparison. Then, the value of index $\mathrm{j}$ in Logistics Equipment System of Systems i could gain its correlation coefficient $c_{i, j}$ according to the following formula:

$$
c_{i, j}=\frac{\min _{i} \min _{j}\left|r_{i, j}-r_{i}^{*}\right|+\xi \times \max _{i} \max _{j}\left|r_{i, j}-r_{i}^{*}\right|}{\left|r_{i, j}-r_{i}^{*}\right|+\xi \times \max _{i} \max _{j}\left|r_{i, j}-r_{i}^{*}\right|}
$$

Where, $\min _{i} \min _{j}\left|r_{i, j}-r_{i}^{*}\right|$ represents the minimum difference, and $\max _{i} \max _{j}\left|r_{i, j}-r_{i}^{*}\right|$ is the maximum difference. $\xi \in(0,1)$ is called Differentiation Coefficient and generally, when $x=0.5$, high resolution could be achieved. In this way, correlation matrix can be attained:

$$
C=\left(\begin{array}{ccc}
c_{11} & \cdots & c_{17} \\
\vdots & \ddots & \vdots \\
c_{n 1} & \cdots & c_{n 17}
\end{array}\right)
$$

\subsection{Weighting Approach based on Information Entropy and AHP}

Without weights determined by decision-makers or experts, the amount of informationcontained in each index could be applied effectively to differentiate the resolution of the planning schemes for assessment. Based on the definition of Entropy introduced in Information Theory,the Information Entropy outputted from the standardized index $r_{i, j}$ is shown as the following formula:

$$
S_{j}=-\frac{1}{\ln n} \sum_{i=1}^{n} r_{i, j} \ln r_{i, j}
$$

When $r_{i, j}=0$, it is ruled that $r_{i, j} \ln r_{i, j}=0$ should be tenable. The following formula can provide a method to calculate the weights of indices:

$$
w_{j}=\frac{1-S_{i}}{\sum_{i=1}^{17}\left(1-S_{i}\right)}
$$

When weights can be determined by decisionmakers or experts, the relative importance of each index is obtained based on which judgment matrix is formed as follow:

$$
U=\left(\begin{array}{ccc}
u_{1,1} & \cdots & u_{1,17} \\
\vdots & \ddots & \vdots
\end{array}\right)
$$

Where, $u_{i, j}$ represents the importance of $u_{i}$ compared touj, and the weights of indices are calculated using Root Method (Griendling\&Mavris 2011). Thecalculation is performed by the following steps:

Multiple all the values in a row to obtain

$$
u_{i}=\prod_{j}^{17} u_{i, j}
$$

(2) Calculate the m root of $u_{i}$ :

$$
\bar{u}_{i}=\sqrt[17]{u_{i}}
$$

(3) Normalize $\bar{u}=\left\{\bar{u}_{1}, \bar{u}_{2}, \ldots \bar{u}_{17}\right\}$ by $w_{i}=\bar{u}_{i} / \sum_{i=1}^{17} \bar{u}_{i}$, and $W=\left(w_{1}, w_{2}, \ldots w_{17}\right)$ turns out to be the final vector of weights satisfying $\sum_{i=1}^{17} w_{i}=1$.

As the last step, all the effectiveness values of Digital Logistics Equipment System of Systems are calculated using the following formula of Grey Hierarchy Correlation Judgment Model:

$$
E=W \times C=\left\{e_{i} \mid e_{i}=\sum_{j=1}^{17} w_{j} c_{i, j}, i=1,2, \ldots . n\right\}
$$

In practical application, the effectiveness values of Digital Logistics Equipment System of Systems can be regarded as the bottom indices correlation and indices values in a higher level can be calculated using the calculation results of the lower level indices, and then the correlation degree in the higher level is attained. By using this algorithm repeatedly in all the levels, the correlation of top level is achieved, which is just the integrated effectiveness value of Digital Logistics Equipment System of Systems and the order of the correlation degree of top level ranks accordingly merits of these system of systems.

\section{CONCLUSION}

Digital Logistics Equipment System of Systems is a huge complex system(Zhang 2010), and its effectiveness can be evaluated using many approaches, each having its own merits. In this paper, the concept of Grey Correlation Degree and Information Entropy are synthetically applied to accomplish the evaluation. The effectiveness evaluation of Digital Logistics Equipment System of Systems provide an auxiliary decision-making approach for the demonstration, research and manufacture of system, and a decisionmaking planning of system construction. On the other hand, this work stimulates the effectiveness enhance and optimization of current system and provides a scientific basis for the research and manufacture of the subsequent system.

The target system and the evaluation mechanism built in this research form a significant reference for the evaluation of other information system, so this method possesses practical value. Furthermore, the approach in this article not only conducts comprehensive quantitative analysis and synthetic evaluation of Digital Logistics Equipment System of Systems, but can be applied in other similar systems.

Indeed, there are also some limitations of this approach. Improvements can be made for future studies in the following ways: First, more attention should be given to the universal application of the weighted average model in the effectiveness computation of each level index, and the effectiveness aggregation model 
can be more flexible and innovative. Second, we will investigate the different weighted methods of effectiveness indices on how to influence the final effectiveness results. Finally, the order of the correlation degree of top level ranks accordingly merits of this system of systems can obtained by more exact and complete decision methods; thus, it is necessary to add experts' judgments to ranking process.

\section{REFERENCE}

Barcio, B.T.,Ramaswamy, S., MacFadzean, R., Barber, K.S., Object-oriented analysis, modeling, and simulation of a national air defense system[C].Systems, Man and Cybernetics, 1995.Intelligent Systems for the 21st Century, IEEE International Conference on, vol.5, no., pp.39833988 vol.5, 22-25 Oct 1995.

BiltgenP.T.,Mavris D.N., Capability-Based Quantitative Technology Evaluation for Systems-of-Systems[C].System of Systems Engineering, 2007.SoSE '07. IEEE International Conference on, vol., no., pp.1-6, 16-18 April 2007.

Boardman J., Pallas S., Sauser B.J., et al,Report on System of Systems Engineering, Final Report for the Office of Secretary of Defense[R]. Stevens Institute of Technology, Hoboken, NJ. http://www.boardmansauser.com / words/ provocations. html Jun 30, 2011.

Chairman of the Joint Chiefs of Staff,Operation of the Joint Capabilities Integration and Development System (CJCS).CJCSI 3170.01G, March 1, 2009.

DeLaurentisD.A., A taxonomy-based perspective for systems of systems design methods[C].Systems, Man and Cybernetics, 2005 IEEE International Conference on, vol.1, No., pp. 86- 91 Vol. 1, 10-12 Oct. 2005.

Evans,R.C.,National Air Defense: Challenges, Solution Profiles, and Technology Needs. The MITRE Corporation [EB/OL]. http://www.mitre.org/work/tech_papers/tech_ papers_04/04_1108/04_1108.pdf. Jun 30, 2011.

Griendling K.,Mavris D.N., Development of a DoDAF-based executable architecting approach to analyze system-ofsystems alternatives[C].Aerospace Conference, 2011 IEEE, vol., no., pp.1-15, 5-12 March 2011.

Ishibuchi H., Tanaka H.,Multiobjective programming in optimization of the interval objective function [J]. European Journal of Operational Research, 48: pp.219-225, 1990.

Joint Systems and Analysis Group of TTCP, Guide to Capability-Based Planning, Technical Cooperation Program Technical Report[R]. TR-JSATP2-2-2004, 2004, pp. 3-6.

Lizotte M.,Bernier F., Mokhtari M., et al,CapDEM-Toward a Capability Engineering Process: A Discussion Pa$\operatorname{per}[R]$. Report No.(s): ADA440003,Dec. 2004.

Maier M.W.,Architecting Principles for Systems-of-Systems [J].Systems engineering, vol 1, No 4, pp 267-84, 1998.

Ramon E. Moore, R. Baker Kearfott, Michael J. Cloud,Introduction to interval analysis [M].Published 2009 by Society for Industrial and Applied Mathematics in Philadelphia.

Sage A.P., CuppanC.D.,On the Systems Engineering and Management of Systems of Systems and Federations of Systems [J]. Information, Knowledge, Systems Management, Vol. 2, No. 4, 2001, pp. 325-45.
Xudong Miao,Demin Xu,Yongchun Wang, Intuitionistic Fuzzy Likelihood Methods and Models for Naval Air Defense Situation and Threat Assessment [J].Fuzzy Systems and Knowledge Discovery, 2009.FSKD '09. Sixth International Conference on, vol.6, no., pp.311-315, 14-16 Aug. 2009.

Zhang Chengbin, Luan Liqiu, Zhang Hongzhou,Multi-agent technology based design of hierarchy model of groups/teams in Air Defense System[C].Audio Language and Image Processing (ICALIP), 2010 International Conference on, vol., no., pp.1403-1406, 23-25 Nov. 2010. 